\title{
Electrophoresis of Protein Charge Ladders: A Comparison of Experiment with Various Continuum Primitive Models
}

\author{
Stuart A. Allison, ${ }^{* \dagger}$ Jeffrey D. Carbeck, ${ }^{\ddagger}$ Chuanying Chen, ${ }^{\dagger, \S}$ and Felicia Burkes ${ }^{\dagger}$ \\ Department of Chemistry, Georgia State University, Atlanta, Georgia 30303, and Department of Chemical \\ Engineering, Princeton University, Room A319, E-Quad, Princeton, New Jersey 08540
}

Received: October 31, 2003; In Final Form: January 19, 2004

\begin{abstract}
Detailed modeling of the free solution electrophoresis of five proteins (bovine $\alpha$-lactalbumin, hen egg white lysozyme, bovine superoxide dismutase, human carbonic anhydrase II, and hen ovalbumin) is carried out within the framework of the continuum primitive model. Protein crystal structures and translational diffusion constants are used to design and parametrize the models. The modeling results are compared with experimental mobilities of protein charge ladders, collections of protein derivatives where the number of charge groups is varied by partial acylation of lysine residues or by amidation of glutamic and aspartic acid residues. The simplest model considered is the Yoon and Kim model of a prolate/oblate ellipsoid with uniform surface potential, electrostatics treated at the level of the linear Poisson-Boltzmann equation, and distortion of the ion atmosphere from equilibrium (ion relaxation) ignored (Yoon, B. J.; Kim, S. J. Colloid Interface Sci. 1989, 128, 275). This model provides good agreement with experiment but only if the net absolute protein charge is low or the average absolute surface, or $\xi$, potential is less than $\sim 25 \mathrm{mV}$. Boundary element (BE) modeling is also carried out in which detailed surface models are employed and the electrostatics are solved at the level of the nonlinear Poisson-Boltzmann equation. Ion relaxation is also included in some of the BE studies. All of the experimental mobilities are in good (human carbonic anhydrase II and hen ovalbumin) to excellent (bovine $\alpha$-lactalbumin, hen egg white lysozyme, and bovine superoxide dismutase) agreement with $\mathrm{BE}$ modeling that includes ion relaxation. We believe that these results taken as a whole serve to confirm the ability of the continuum primitive model to predict, with quantitative accuracy, the free solution electrophoretic mobilities of proteins, provided the underlying models are sufficiently realistic. When a discrepancy occurs, it may be due to error in modeling either the protein charge or the solution conformation. The models described in this work also provide a useful approach for determining values of $\Delta Z$, the change in net charge of proteins due to the chemical modification of charged groups. Knowledge of $\Delta Z$ is essential for the use of protein charge ladders in the quantitative description of the electrostatic properties and interactions of proteins. This paper supports the view that the continuum primitive model may be more appropriate for the modeling of electrokinetics than for electrostatics. The main challenge to the accurate predictions of electrophoretic mobilities may lie primarily in the modeling of electrostatics, not electrokinetics.
\end{abstract}

\section{Introduction}

The prediction of electrophoretic mobilities of proteins from their sequences and structures is challenging because such predictions require accurate models of both the electrostatic and the electrokinetic properties of proteins. Electrostatic models are used to predict the distribution of charges on the protein due to the reversible binding of protons to ionizable groups and also the electrostatic potential in the vicinity of the model protein. Electrokinetic models take as input a particular distribution of charge and electrostatic potential and predict the electrophoretic mobility. Both types of models are generally based on the continuum primitive model. The combination of capillary electrophoresis (CE) and protein charge ladders is a useful experimental system for addressing this challenge, as it provides values of electrophoretic mobilities for a collection of

* To whom correspondence should be addressed. Phone: (404)651-1986. E-mail: chesaa@panther.gsu.edu.

Georgia State University.

$\doteqdot$ Princeton University.

$\S$ Current address: Department of Chemistry, University of Houston, Houston, TX 77004. protein derivatives that differ incrementally in the number of ionizable groups. In the prediction of electrophoretic mobilities of protein charge ladders, most of the effort has been devoted to modeling of the electrostatics; only simple models of the electrokinetics have been applied. The objective of this paper is to compare experimental mobilities with several electrokinetic models and to determine the degree to which these models can accurately predict the electrophoretic mobilities of proteins.

The combination of $\mathrm{CE}$ and charge ladders has been used for the characterization of a variety of physical properties and interactions of proteins: examples include their net charges, ${ }^{1}$ hydrodynamic sizes, ${ }^{2}$ molecular weights, ${ }^{3}$ dissociation of titratable groups,,${ }^{4,5}$ and stabilities. ${ }^{6}$ Charge ladders are produced by the partial and chemical modification of charged groups on the protein. The most common procedures involve the acylation of lysine residues with acetic anhydride ${ }^{3}$ or the conversion of aspartic and glutamic acid residues to hydroxamic groups (amidation) with hydroxylamine. ${ }^{7}$ In free solution capillary electrophoresis, these modified proteins separate into distinct bands, or "rungs", in which each rung consists of species with 
the same number of chemical modifications due to similarities in their net charges. ${ }^{1-7}$

As investigators have attempted to extract more quantitative information from these charge ladder studies, such as the average charge difference, $\Delta Z$, between successive rungs of a ladder, it has become apparent that more realistic modeling of the free solution electrophoresis of proteins is needed. Up to the present, much of the analysis has been based on the Henry model, ${ }^{8}$ in which the protein is modeled as a solid sphere with a centrosymmetric charge distribution. The surrounding fluid is modeled as a hydrodynamic continuum with viscosity $\eta$ and dielectric constant $\epsilon_{\mathrm{r}}$. In addition, mobile ions in the solvent are modeled as a continuum and local ion concentrations are assumed to depend on the equilibrium electrostatic potential by the Poisson-Boltzmann equation. In terms of this model, there are two parameters: the protein radius, $R$, and the net protein charge, $Z$ (in dimensionless units of the protonic charge).

To estimate $Z$ from the charge ladder studies, it was initially assumed that $\Delta Z=-1$ for the acylation of lysines. However, neutralization of charge residues may alter the charge state of neighboring sites; this phenomenon, referred to as "charge regulation", can cause $|\Delta Z|$ to be smaller than $1 .^{9}$ Charge regulation has been examined in terms of the Linderstrom-Lang model, ${ }^{10}$ which employs a spherical model identical to that of the Henry model of electrophoresis ${ }^{8}$ and estimates the fractional dissociation of specific charge groups on the protein surface and hence the net protein charge. Acylation of a particular lysine residue would not be expected to affect the charge state, or $\mathrm{p} K_{\mathrm{a}}$, of all residues of the same type (histidines, for example) to the same extent. Histidines closer to the acylation site should experience a stronger shift in $\mathrm{p} K_{\mathrm{a}}$ than histidines that are further away. Over the last 15 years, atomically detailed models have been used to compute the charge states of proteins at the level of the linear Poisson-Boltzmann equation. ${ }^{11-15}$ These methods have been used in the analysis of the free solution electrophoresis of protein charge ladders. ${ }^{16,17}$

Proteins typically are irregular in shape, carry complex charge distributions, and are not large compared to the thickness of the ion atmosphere that surrounds them. There is a need, therefore, to consider electrophoretic models that account more accurately for the detailed shapes and charge distributions of proteins. An important question we shall address is how much "detail" in models is necessary in order to obtain good agreement between experiment and theory. The Yoon and Kim model, ${ }^{18}$ which models the protein as a prolate or oblate ellipsoid with a uniform surface potential, shall be examined. As in the case of the Henry model, the equilibrium charge distribution of ions in the vicinity of the model protein is employed. In addition, the Poisson-Boltzmann equation is solved at the linear level. For more detailed accounting of protein shape and charge distribution, boundary element (BE) methods shall be used in this work. ${ }^{19-21}$ It should be pointed out, however, that BE methods are not yet widely available and are computationally expensive to carry out. In addition to accounting for detailed shape and charge distribution, account can be taken of "ion relaxation", which refers to the distortion of the local ion distribution from equilibrium due to the presence of an external electric or flow field. ${ }^{22,23}$ This effect is important for highly charged macroions including certain portions of the charge ladders of proteins. All of the models and methodologies discussed above are grounded on the continuum primitive model $(\mathrm{CPM}) .{ }^{24}$ In the CPM, the particle is represented as an arbitrary low dielectric rigid body with an arbitrary, but defined, charge distribution within and/or on the particle. Outside the particle- solution interface, the local viscosity changes from a high value to that of the bulk solution over a narrow region; this region is contracted to a smooth surface-called the hydrodynamic shear surface, $S_{\mathrm{p}}$ - that envelopes the particle. Outside of $S_{\mathrm{p}}$, the solvent is represented as an incompressible fluid of viscosity $\eta$ and dielectric constant $\epsilon_{\mathrm{r}}$. Mobile ions are treated as a continuum as well, and the charge distribution is assumed to obey the Poisson-Boltzmann (equilibrium) or Poisson (nonequilibrium) equation.

One of the advantages of detailed BE modeling is that it should be possible to determine if the CPM is capable of accurately predicting the free solution electrophoretic mobility of proteins. If the answer to this question is yes, a second question is how sensitive are electrophoretic mobilities to details of protein structure and charge? We shall attempt to answer both of these questions by modeling the electrophoretic mobilities of charge ladders of five proteins reported in the literature.

\section{Methodology}

A. Analytical Models. The model for electrophoresis we should mention first is that of Henry, ${ }^{8}$ which treats the protein or macroion as a sphere of radius $R$ containing a centrosymmetric charge distribution of net charge $q Z\left(q=1.602 \times 10^{-19}\right.$ C) in a salt solution with Debye-Hückel screening parameter $\kappa$. (More precisely, the surface of hydrodynamic shear, $S_{\mathrm{p}}$, is modeled as a sphere of radius $R$.) The distortion of the ion atmosphere in the vicinity of the macroion from equilibrium, or ion relaxation, is ignored. Neglect of ion relaxation is a good approximation provided the absolute electrostatic potential at the macroion surface does not exceed $\sim 25 \mathrm{mV} .^{25}$ This model has been used in the past to interpret the electrophoretic mobility of protein charge ladders. In the present work, however, we shall discuss the more general Yoon and Kim model, ${ }^{18}$ which treats the mobility of a prolate or oblate spheroidal particle with uniform surface potential, $\psi_{0}$, at the solvent-particle interface. Let $a$ and $c$ denote the semi-axes of the ellipsoid with $a \geq c$. The orientationally averaged mobility, $\mu$, reduces to that of the Henry model in the limit of a sphere with $a=c=R$. As in the case of the Henry model, ion relaxation is ignored and the ion distribution is assumed to obey the Poisson-Boltzmann (PB) equation

$$
\nabla^{2} \Lambda_{0}=-\frac{\rho_{0}\left(\Lambda_{0}\right)}{\epsilon_{0} \epsilon_{\mathrm{r}}}=-\frac{1}{\epsilon_{0} \epsilon_{\mathrm{r}}} \sum_{i} n_{i} \bar{z}_{i} q e^{-q z_{i} \Lambda_{0} / k_{\mathrm{B}} T}
$$

where $\Lambda_{0}$ is the equilibrium electrostatic potential at some point in the fluid domain near the model macroion, $\epsilon_{0}$ is the permittivity of free space, $\epsilon_{\mathrm{r}}$ is the solvent dielectric constant, $\rho_{0}$ is the local charge density, the sum over $i$ is over all mobile ion species present, $n_{i}^{\circ}$ is the bulk concentration of species $i, z_{i}$ is the valence of species $i, k_{\mathrm{B}}$ is Boltzmann's constant, and $T$ is the absolute temperature. At low surface potential $\left(q \Lambda_{0} / k_{\mathrm{B}} T \ll\right.$ $1)$, eq 1 reduces to the linear $\mathrm{PB}$ equation

$$
\nabla^{2} \Lambda_{0}=\kappa^{2} \Lambda_{0}
$$

where

$$
\kappa=\sqrt{\frac{q^{2} \sum_{i} n_{i}^{0} z_{i}^{2}}{\epsilon_{0} \epsilon_{\mathrm{r}} k_{\mathrm{B}} T}}
$$

In water at $25^{\circ} \mathrm{C}$ containing monovalent salt of net positive or 
negative ion concentration equal to $I \mathrm{~mol} / \mathrm{L}, \kappa($ in $1 / \mathrm{nm})=$ $3.288 I^{1 / 2}$. For an ion distribution which satisfies eq 2, Yoon and Kim obtained ${ }^{18}$

$$
\mu_{\mathrm{YK}}=\frac{\epsilon_{0} \epsilon_{\mathrm{r}} \psi_{0}}{\eta} C_{\mathrm{Av}}(\kappa c)
$$

where $C_{\mathrm{Av}}$ is a slowly varying function of $\kappa c$ summarized in Figure 5 of ref 18 . In the limit of a sphere, $C_{\mathrm{Av}}$ reduces to Henry's function, $f(\kappa c)$. It is important to note that $C_{\mathrm{Av}}$ is relatively insensitive to particle shape as well as size and goes to $2 / 3$ in the limit $\kappa c \rightarrow 0$ and 1 as $\kappa c \rightarrow \infty$. For proteins at the ionic strength of interest in this work, $\kappa c \approx 1$ and $C_{\mathrm{Av}}(\kappa c)$ lies close to its lower bound value of $2 / 3$. Consequently, it is convenient to define

$$
g(\kappa c)=\frac{3}{2} C_{\mathrm{Av}}(\kappa c)
$$

where $3 / 2>g(\kappa c)>1$ in general but $g(\kappa c) \approx 1.0$ for the proteins of interest in this work. It is also possible to express $\psi_{0}$ in terms of the net charge within/on the ellipsoid, $q Z$,

$$
\psi_{0}=\frac{q Z}{4 \pi \epsilon_{0} \epsilon_{\mathrm{r}} R^{*}\left(1+\kappa R^{*}\right)}
$$

where $R^{*}$ is an effective particle size related in a straightforward way to the dimensions of the ellipsoid:

$$
R^{*}=\frac{2 a d}{\ln \left(\frac{1+d}{1-d}\right)} \quad \text { (prolate) or } \frac{a d}{\cot ^{-1}\left(\frac{\sqrt{1-d^{2}}}{d}\right)} \text { (oblate) }
$$

where $d=\left(1-c^{2} / a^{2}\right)^{1 / 2}$ is the eccentricity of the ellipsoid. ${ }^{18}$ Using eqs 5 and 6 in eq 4 gives eq 8:

$$
\mu_{\mathrm{YK}}=\frac{q Z g(\kappa c)}{6 \pi \eta R^{*}\left(1+\kappa R^{*}\right)}
$$

If $\mu$ is in $\mathrm{cm}^{2} /(\mathrm{kV} \mathrm{s})$ (units used throughout this work), $\eta$ is in centipoise, and $R^{*}$ is in $\mathrm{nm}$, then eq 8 reduces to

$$
\mu_{\mathrm{YK}}=\frac{A Z g(\kappa c)}{\eta R^{*}\left(1+\kappa R^{*}\right)}
$$

where $A=0.08499$. The advantage of eqs 8 and 9 is their simplicity. Except for the inclusion of an additional parameter (the particle radius, $R$, being replaced with $R^{*}$, which, in turn, is a simple function of $a$ and $c$ ), the basic form of the Henry model is retained.

In the capillary electrophoresis of protein charge ladders, it is observed that the proteins migrate in bands. On the basis of the Henry and Yoon and Kim models, these bands correspond to proteins of equal size, shape, and charge. Let $Z_{0}$ denote the charge of the unmodified protein, $n$ the number of modified charge residues, and $\Delta Z$ the average change in protein charge that occurs when a single residue is modified. Also let $\mu_{0}$ and $\mu_{n}$ represent the (low field) free solution electrophoretic mobilities of the unmodified protein and of the protein with $n$ modified charge residues, respectively. Assuming the validity of eq 9 and that protein conformation does not vary with $n$

$$
\mu_{n}=\mu_{0}+s n
$$

where $s=\mathrm{d} \mu / \mathrm{d} n$ and

$$
\begin{gathered}
\mu_{0}=\frac{A Z_{0} g(\kappa c)}{\eta R^{*}\left(1+\kappa R^{*}\right)} \\
s=\frac{A \Delta Z g(\kappa c)}{\eta R^{*}\left(1+\kappa R^{*}\right)}
\end{gathered}
$$

If a value of $\Delta Z$ is assumed and if the macroion is also assumed to be spherical, it is straightforward to extract both $R^{*}(=R)$ and $Z_{0}$ from a charge ladder electrophoresis experiment. However, because of the charge regulation discussed briefly in the Introduction, $\Delta Z$ may depart from \pm 1 , depending on a variety of factors such as the $\mathrm{pH}$ and ionic strength as well as the nature of the particular protein being investigated. Assuming the protein is spherical is clearly an approximation as well.

In many cases, there are independent experimental data available that are useful in modeling protein electrophoresis. Sedimentation or diffusion constants have been reported for many proteins in both handbooks ${ }^{26}$ and the literature. For example, the translational diffusion constant, $D_{\mathrm{t}}$, provides information about particle size. For a prolate or oblate ellipsoid, $D_{\mathrm{t}}$ is given $\mathrm{by}^{27}$

$$
D_{\mathrm{t}}=\frac{k_{\mathrm{B}} T}{6 \pi \eta R^{*}}
$$

where $R^{*}$ is given by eq 7. In addition, the Protein Data Bank contains crystal structures for a vast array of biomolecules and biomolecular complexes that are readily accessible on the Internet. If a crystal structure is available, it is possible to make reasonable assumptions about the shape and size of the protein (or other biomolecule/biomolecular complex) in solution. For the proteins considered in this work, crystal structures are available and, in most cases, so are diffusion constants. From the atomic coordinates, the protein is placed in a reference frame whose origin is at the center of mass and also diagonalizes a tensor similar to the moment of inertia tensor. ${ }^{28,29}$ From the maximum and minimum atom positions in the $x, y$, and $z$ directions in this reference frame, it is possible to identify a plausible ellipsoidal shape of a particular protein, specifically $p=a / c$. Equations 7 and 13 can then be used to determine what $a$ and $c$ must be employed to yield the value of $D_{\mathrm{t}}$ observed experimentally. At this point, eqs 11 and 12 contain only two unknowns, $Z_{0}$ and $\Delta Z$. From $\mu_{0}, Z_{0}$ is determined, and from the slope, $s, \Delta Z$ is determined.

There are three shortcomings of this approach. (I) It is based on a model in which the protein is assumed to be prolate or oblate ellipsoidal in shape with a uniform surface potential, $\psi_{0}$. From crystal structures, it is clear that protein topography is more complicated than this. (II) The surface potentials of proteins are not uniform, since charge is localized on specific residues. In principle, variation in potential on the surface of a particle does influence mobility, particularly if the particle is nonspherical. ${ }^{30}$ (III) It is readily observed from a number of charge ladder studies that a plot of mobility versus $n$ becomes progressively more nonlinear as the net absolute charge of the protein increases. The Yoon and Kim model predicts a linear dependence of mobility on net charge.

There are three reasons why the Yoon and Kim model does not predict the observed nonlinear correlation between mobility and $n$. First, it is based on the solution of the linear PB equation (eq 2). For highly charged proteins, the full $\mathrm{PB}$ equation is more appropriate (eq 1). Second, for highly charged proteins, ion relaxation becomes increasingly important and it is ignored in the Henry ${ }^{8}$ and Yoon and Kim ${ }^{18}$ models. The effects of ion 

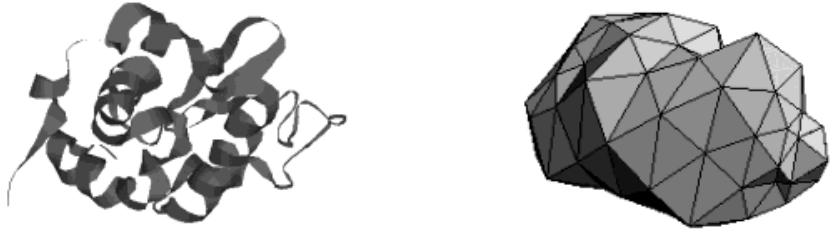

Figure 1. Ribbon and BE plate model for bovine $\alpha$-lactalbumin. The crystal structure is from Protein Data Bank file 1F6S. The BE model consists of 144 plates and is designed to (a) conform closely with the crystal structure and (b) yield the translational diffusion constant, $D_{\mathrm{t}}$, measured experimentally.

relaxation on the electrophoresis of spheres, ${ }^{22,23,31,32}$ cylinders, ${ }^{33,34}$ and arbitrary model particles not large relative to $1 / \kappa^{19-21}$ have been examined. Modeling proteins as spheres showed that the effects of ion relaxation and the use of the full PB equation could account for much of the nonlinearity observed for the charge ladder of protein human carbonic anhydrase. ${ }^{2}$ Third, the proteins could be undergoing a conformational change as the net absolute charge becomes large. Thus, the effects of complex shape/charge distribution as well as the first two causes of nonlinear charge effects can be considered and this is done in the present work. The possibility of a conformational change shall also be addressed indirectly by observing how well rigid model studies are able to reproduce the experimental mobilities of charge ladders.

B. Boundary Element Methodology. The boundary element (BE) methodology for determining transport properties of rigid model particles has been discussed in detail previously. ${ }^{19-21}$ The electrostatic and electrodynamic potentials are obtained by numerical solution of the nonlinear $\mathrm{PB}$ and general Poisson equations. Potential functions related to the distortion of the ion distributions from equilibrium, ion relaxation, are obtained by solution of a steady state ion transport equation for each of the mobile ion species present. The fluid velocity in the vicinity of the model particle is also needed, which requires solution of the low Reynolds number Navier-Stokes equation for an incompressible fluid. Under conditions where ion relaxation is significant, these various field equations are coupled together and it is necessary to solve them by an iterative procedure. ${ }^{19}$

The model protein is represented as a plated structure, and an example of the protein $\alpha$-lactalbumin is shown in Figure 1 along with a ribbon structure. The coordinates are "downloaded" from the Protein Data Bank, and they are then rotated to orient the particle so that its principal axis is along the $x$ axis. The method used in the present work to generate the plated structures follows very closely the procedure reported previously in the construction of detailed models of short duplex DNA fragments. ${ }^{35}$ As discussed in that work, a "protocylinder" is first constructed with the long axis in the $x$ direction. The length of the protocylinder and its axial radius are chosen to entirely enclose all atoms. However, the $y$ and $z$ positions of the "origin vectors" are allowed to vary and are selected to correspond to a local center of mass. Then, an "effective atom exclusion radius", $\sigma$, is chosen and the protocylinder is "deflated" until all vertex vectors of the plates fall at a distance $\sigma$ relative to the nearest atom of the entire protein. That value of $\sigma$ which reproduces $D_{\mathrm{t}}$ is then used to define the hydrodynamic shear surface, $S_{\mathrm{p}}$. (In the case of bovine superoxide dismutase, $D_{\mathrm{t}}$ is not known to us. Values are reported in the literature ${ }^{36}$ but undoubtedly correspond to aggregates. For that case, $\sigma$ is set to $0.45 \mathrm{~nm}$, which is a typical value for proteins modeled by this procedure.) As discussed previously, it is also necessary to construct a series of fluid shells increasing in thickness moving away from $S_{\mathrm{p}}$. This is achieved by constructing 50 surfaces in a manner identical to the procedure described previously but by increasing $\sigma$. The final surface is placed at a distance $b / \kappa$ from $S_{\mathrm{p}}$, where $b$ is typically $12-16 .^{37}$

Once a plated structure and its shell array are constructed, the orientationally averaged translational diffusion constant, $D_{\mathrm{t}}$, and the electrophoretic mobility, $\mu$, as well as other transport properties, ${ }^{38}$ are obtained by the BE procedure. For an irregularly shaped protein model, six separate BE calculations are carried out. In the first three (case 1), the model protein is placed in a constant external flow field directed along three orthogonal directions. In the second three (case 2), the model protein is placed in a constant external electric field directed along three orthogonal directions. From the total forces acting on the model particle in these six calculations, friction and "tether force" tensors are derived. From these tensors, straightforward averaging yields $D_{\mathrm{t}}$ and $\mu$. $^{20}$

C. Estimating Protein Charge. An essential element in the design of models of the electrophoretic mobility of proteins is the charge distribution, which arises from the protonation/ deprotonation of ionizable residues. For the proteins considered in this work, detailed crystal structures are available and there is no question about the identity and position of ionizable sites. In a few cases such as hen egg white ${ }^{39,40}$ and T4 lysozyme, ${ }^{41}$ the $\mathrm{p} K_{\mathrm{a}}$ 's of the ionizable residues are, for the most part, known. (In the present work, the $\mathrm{H}^{+}$concentrations referred to by the $K_{\mathrm{a}}$ 's are the bulk ion concentrations rather than the local ion concentrations.) Thus, there is little uncertainty of the degree of ionization of a particular residue and hence the net protein charge at a particular $\mathrm{pH}$. In the past, $\mathrm{BE}$ modeling of unmodified hen egg white lysozyme versus $\mathrm{pH}^{42}$ and of unmodified plus charge mutants of T4 lysozyme ${ }^{43}$ has been carried out. For most proteins, including four of the five reported in this work, the values of $\mathrm{p} K_{\mathrm{a}}$ of individual residues are not known, although standard values, $\mathrm{p} K_{\mathrm{a} i}^{\circ}$, are available. ${ }^{7}$ These $\mathrm{p} K_{\mathrm{a} i}^{\circ}$ 's refer to the ion dissociation constants of specific groups in an environment of zero electrostatic potential on the protein surface relative to the bulk solution. In this work, these "standard values" are taken to be $\operatorname{Arg}=12, \operatorname{Lys}=10.7, \mathrm{Tyr}=10.2$, hydroxamic acid $=10$, Cys $=9.3, \alpha-\mathrm{NH}_{3}{ }^{+}=7.4$, His $=6.5$, $\alpha-\mathrm{COOH}=4.9$, Glu $=4.4$, and $\mathrm{Asp}=4.0$.

Over the last 10 years, numerical methods have been developed to determine the $\mathrm{p} K_{\mathrm{a} i}$ 's of protein residues within the framework of the linear PB equation (eqs 11-15). Recently, Sharma et al. applied this approach in a study of the charge ladder of hen egg white lysozyme and showed that it effectively identifies both specific and nonspecific cooperative interactions between titratable groups. ${ }^{17}$ Sharma et al. went on to show that a simpler empirical method, based on the approach of Lee et al., ${ }^{44}$ effectively describes the cooperativity in proton binding. Consider residue $i$ on the surface of a protein in a solvent with dielectric constant $\epsilon_{\mathrm{r}}$ and Debye-Hückel screening parameter $\kappa$. Let $\Delta \psi_{i, j}$ denonte the potential at site $i$ due to a charge, $q \Delta z_{j}$ $\left(q=1.602 \times 10^{-19} \mathrm{C}\right)$, being placed at ionizable site $j$. The change in $\mathrm{p} K_{\mathrm{a} i}, \Delta \mathrm{p} K_{\mathrm{a} i, j}^{\circ}=\mathrm{p} K_{\mathrm{a} i}^{\circ}-\mathrm{p} K_{\mathrm{a} i}^{\circ}$, due to this perturbation is

$$
\Delta \mathrm{p} K_{\mathrm{a} i, j}=-\frac{q \Delta \psi_{i, j}}{2.303 k_{\mathrm{B}} T}=-\frac{q^{2} \Delta z}{2.303 \epsilon_{\mathrm{r}} k_{\mathrm{B}} T} \frac{\mathrm{e}^{-\kappa r_{i j}}}{r_{i j}}
$$

where $r_{i j}$ is the distance between ionizable sites $i$ and $j$. The uncertainty in the degree of ionization of a particular residue can be minimized by working at a $\mathrm{pH}$ that is far removed from $\mathrm{p} K_{\mathrm{a} i}^{\circ}$. Although $\Delta \mathrm{p} K_{\mathrm{a} i, j}^{\circ}$ may be significant, the degree of ionization of $i$ is not significantly affected under these circum- 
stances. In the present work, all studies are done at $\mathrm{pH}=8.4$ and this minimizes the uncertainty in the degree of ionization of most residues and consequently the net protein charge. The $\mathrm{p} K_{\mathrm{a} i}^{\circ}$ values listed at the end of the previous paragraph are used along with eq 14 . The revised $\mathrm{p} K_{\mathrm{a} i}$ 's must be solved for iteratively. Initially, we set $\mathrm{p} K_{\mathrm{a} i}=\mathrm{p} K_{\mathrm{a} i}^{\circ}$ and compute the degree of ionization of all residues at the assigned $\mathrm{pH}$ (8.4 in this case). Summing eq 14 over all $j \neq i$, a new estimate of each $\mathrm{p} K_{\mathrm{a} i}$ is computed. The revised $\mathrm{p} K_{\mathrm{a} i}$ 's are then used to reestimate the residue charge or degree of ionization. This procedure is repeated until the $\mathrm{p} K_{\mathrm{a} i}$ 's converge to a tolerance of $0.1 \%$. It should be emphasized that this approach is an approximation of a formal calculation of $\mathrm{p} K_{\mathrm{a} i}$ at the level of the linear PB equation (eqs 11-15). However, since $\mathrm{p} K_{\mathrm{a} i}$ 's computed by the formal procedure are, in instances, at some variance with experimental $\mathrm{p} K_{\mathrm{a} i}$ ' $\mathrm{s},{ }^{13,14}$ and since the formal procedure is carried out at the level of the linear PB equation, we believe that this simple procedure is adequate for the present work. It should be emphasized that defining the charge distribution of a model protein is approximate. However, the charge distribution or the $\mathrm{p} K_{\mathrm{a} i}^{\circ}$ 's are in no way treated as adjustable parameters to fit mobility data.

The effect of charge regulation is included in our model studies. For each member of the charge ladder, the iteration procedure discussed above is carried out. For example, suppose a particular lysine residue is acylated. Instead of simply setting the charge on that residue to zero, only the intrinsic charge on that residue is set to zero. Then, the iteration procedure of eq 14 is carried out. The net protein charge may change by a value different than -1.00 , since the value of $\mathrm{p} K_{\mathrm{a} i}$ and, thereby, the degree of ionization of sites near the acylated lysine may be affected.

D. Estimating $\Delta \boldsymbol{Z}$. As discussed in section II.A, the Yoon and Kim model can be reduced to a form (eq 9) that is directly proportional to the net charge contained on or within the model protein. It should be emphasized, however, that this is an approximation. In general, the mobility will depend on the charge distribution as well. Perhaps the most convincing demonstration of this comes from the work of Fair and Anderson. ${ }^{30}$ In practice, the dependence of mobility on charge distribution appears to be fairly weak on the basis of analytical theory $^{30}$ as well as extensive BE modeling. ${ }^{19-21,35,37}$ In the Results section of this work, we shall examine how the mobility varies within a single rung of a charge ladder for hen ovalbumin. For proteins of identical size, shape, and net charge, but different charge distribution, the variation in mobility is small. Consequently, it is a reasonable approximation to assume the mobility, $\mu$, is a function of the average net charge, $Z$, although that functional dependence is not necessarily a linear one. Let $n$ represent the number of lysine residues acylated (or minus the number of aspartic or glutamic residues amidated). Then we can write

$$
\left\langle\frac{\mathrm{d} \mu}{\mathrm{d} n}\right\rangle \approx\left\langle\frac{\mathrm{d} \mu}{\mathrm{d} Z}\right\rangle\left\langle\frac{\mathrm{d} Z}{\mathrm{~d} n}\right\rangle \approx\left\langle\frac{\mathrm{d} \mu}{\mathrm{d} Z}\right\rangle \Delta Z
$$

It should also be emphasized that the derivatives in eq 15 correspond to differences in mobilities that are averages over conformers that have the same net charge, Z. Now $\langle\mathrm{d} \mu / \mathrm{d} n\rangle$ is obtained directly from experiment. In the present work, $\langle\mathrm{d} \mu /$ $\mathrm{d} Z\rangle$ is derived from BE modeling studies. A plot of $\langle\mathrm{d} u / \mathrm{d} Z\rangle$ from modeling is constructed and fit with a second order polynomial in $Z$. From this polynomial, $\Delta Z$ is estimated as

$$
\Delta Z=\left\langle\frac{\mathrm{d} \mu}{\mathrm{d} n}\right\rangle_{\mathrm{expt}} /\left(\frac{\mathrm{d} \mu}{\mathrm{d} Z}\right\rangle_{\mathrm{BE}}
$$

Following this procedure, it is possible, in principle, to estimate $\Delta Z$ between successive rungs of the charge ladder. In practice, statistical uncertainty in both experiment and modeling makes an accurate determination of the $n$-dependence of $\Delta Z$ impossible. However, $\Delta Z$ 's averaged over the entire ladder shall be determined.

\section{Results}

Crystal structures for hen egg white lysozyme (2LZT), bovine $\alpha$-lactalbumin, (1F6S), hen ovalbumin (1OVA), human carbonic anhydrase II (1CA2), and bovine superoxide dismutase (1CBJ) are downloaded from the Protein Data Bank. With the exception of superoxide dismutase (SOD), where the solution structure is known to be a dimer, ${ }^{45}$ modeling is carried out on the monomeric forms of the proteins. In SOD, the charge on both $\mathrm{Zn}$ and $\mathrm{Cu}$ atoms is taken to be +2 . For hen ovalbumin, chain $\mathrm{D}$ of the crystal structure is used and the dephosphorylated form of the protein is modeled. ${ }^{46}$ In four of the five proteins, the exception being $\alpha$-lactalbumin, the buffer consists of $25 \mathrm{mM}$ Tris base plus $192 \mathrm{mM}$ glycine at $\mathrm{pH}=8.4 .^{7}$ This corresponds to a salt solution that is $7.9 \mathrm{mM}$ in both Tris ${ }^{+}$and gly $^{-}$. For $\alpha$-lactalbumin, the buffer contains $20 \mathrm{mM} \mathrm{NaCl}$ in addition to the Tris and glycine present in the other solutions. ${ }^{6}$ All modeling studies are carried out at $25^{\circ} \mathrm{C}$, as done in the experiments. ${ }^{6,7}$ $\mathrm{BE}$ models are constructed following the procedure described in section II.B. In most cases, the proteins are modeled as structures made up of 144 plates, and Figure 1 shows an example. Limited modeling studies have been carried out in which the number of plates, $N$, is varied from 96 to 216 . In the present applications, little systematic variation is observed in mobility with $N$ and, consequently, $N$ is set to 144 in all of the modeling studies reported in this work.

An obvious complication in modeling charge ladders is the large number of structures that might correspond to a single rung of the charge ladder. In the amidation of hen ovalbumin, for example, there are 47 different glutamic and aspartic residues in the monomeric protein. If, for example, eight of these are converted to hydroxamic acid residues ${ }^{7}$ and the amidation is approximated to be purely random, there are $3.14 \times 10^{8}$ possible proteins corresponding to the single rung of eight amidation sites. Because of charge regulation, ${ }^{9,47}$ the net protein charge is not expected to be identical in all of these. In addition, protein charge distribution does, in principle at least, influence mobility. In view of these factors, some variation in mobility within a single rung is expected. However, the fact that the free solution electrophoresis of charge ladders yields distinct bands of proteins migrating at different rates leads us to conclude that the variation in mobility/net charge is small relative to the differences in mobility/charge between proteins in different rungs. It is straightforward to address this issue in BE modeling. For the sake of illustration, the amidation and acylation of several rungs of the hen ovalbumin charge ladder are described. The convention used in this work represents the amidation of glutamic and aspartic acid residues with negative rung numbers, $n$, and the acylation of lysines with positive rung numbers. The unmodified protein corresponds to $n=0$. At random, $n$ residues are chosen and amidated or acylated. The charge state of the protein is computed following the procedure described in section II.C. The assumption is made that the conformation of the protein does not change relative to that of the unmodified protein; the $\mathrm{BE}$ approach is used to determine $\mu$. This process is repeated for a 
TABLE 1: Model Variations within the Charge Ladder of Ovalbumin

\begin{tabular}{cccccc}
\hline$n$ & $-Z$ & \multicolumn{1}{c}{$-y_{0}$} & $-\mu(\mathrm{nr})^{a}$ & $-\mu(\mathrm{r})^{a}$ & $\mu(\mathrm{r}) / \mu(\mathrm{nr})$ \\
\hline-8 & $6.04 \pm 0.17$ & $0.840 \pm 0.022$ & $0.1134 \pm 0.0031$ & $0.1101 \pm 0.0031$ & $0.970 \pm 0.003$ \\
-7 & $6.74 \pm 0.18$ & $0.958 \pm 0.011$ & $0.1295 \pm 0.0026$ & $0.1252 \pm 0.0025$ & $0.966 \pm 0.004$ \\
-6 & $7.48 \pm 0.12$ & $1.052 \pm 0.019$ & $0.1408 \pm 0.0056$ & $0.1355 \pm 0.0059$ & $0.963 \pm 0.004$ \\
-5 & $8.40 \pm 0.15$ & $1.175 \pm 0.031$ & $0.1565 \pm 0.0047$ & $0.1498 \pm 0.0045$ & $0.957 \pm 0.003$ \\
-4 & $9.31 \pm 0.08$ & $1.288 \pm 0.010$ & $0.1743 \pm 0.0033$ & $0.1658 \pm 0.0033$ & $0.951 \pm 0.002$ \\
+5 & $17.52 \pm 0.13$ & $2.231 \pm 0.024$ & $0.2994 \pm 0.0046$ & $0.2625 \pm 0.0031$ & $0.877 \pm 0.003$ \\
+6 & $18.46 \pm 0.09$ & $2.350 \pm 0.026$ & $0.3161 \pm 0.0054$ & $0.2744 \pm 0.0047$ & $0.868 \pm 0.003$ \\
+7 & $19.35 \pm 0.06$ & $2.417 \pm 0.025$ & $0.3232 \pm 0.0047$ & $0.2773 \pm 0.0040$ & $0.858 \pm 0.003$ \\
+8 & $20.22 \pm 0.07$ & $2.476 \pm 0.025$ & $0.3310 \pm 0.0056$ & $0.2810 \pm 0.0045$ & $0.849 \pm 0.002$
\end{tabular}

${ }^{a} \mathrm{In}_{\mathrm{cm}^{2}} /(\mathrm{kV} \mathrm{s})$. Also, $\mathrm{nr}$ and $\mathrm{r}$ refer to no ion relaxation and ion relaxation, respectively.

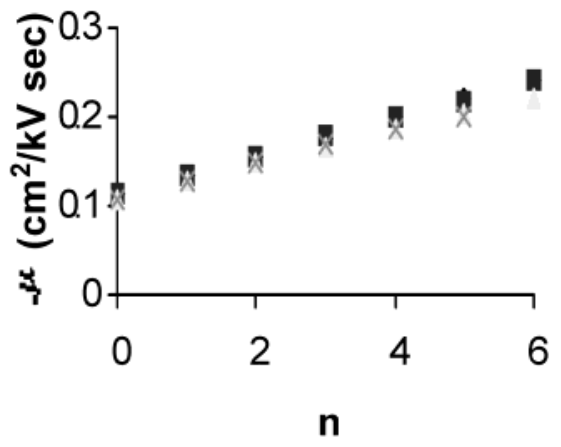

Figure 2. Mobility vs $n$ for bovine $\alpha$-lactalbumin. The studies are carried out in $25 \mathrm{mM}$ Tris base plus $192 \mathrm{mM}$ glycine plus $20 \mathrm{mM}$ $\mathrm{NaCl}$ at $25{ }^{\circ} \mathrm{C}$. Yoon and Kim model results are represented with diamonds, $\mathrm{BE}$ in the absence of ion relaxation is represented with squares, BE including ion relaxation is represented with triangles, and experimental mobilities (6) are represented with $\times$ 's.

total of 4-10 independent runs for each rung of the charge ladder. The results are summarized in Table 1 . In this table, $y_{0}$ $=q\left\langle\Lambda_{0}\right\rangle / k_{\mathrm{B}} T$ represents a dimensionless potential averaged over the protein surface. Also, $\mu(\mathrm{nr})$ and $\mu(\mathrm{r})$ represent the mobilities in the absence and presence of ion relaxation. Standard deviations are included as well. Within a single rung of the charge ladder, the relative variation in $Z$ and $\mu$ is approximately $3 \%$. With regards to mobility, this variation is smaller than the variation in mobility between different rungs of the charge ladder when $|Z|$ is small, but when $|Z|$ is large, the variation in mobility between proteins within a rung becomes comparable to the variation between proteins in different rungs. This effect is not a result of the systematic change in variation within a rung as $|Z|$ increases; the variation in mobility within a rung appears to be independent of $|Z|$, or nearly so. Instead, it is the result of ion relaxation, which reduces the increase in mobility for an incremental increase in $|Z|$ as the value of $|Z|$ becomes large. For the example of ovalbumin considered in Table 1, this occurs for $n \approx+8$. Similar behavior is observed for all of the proteins considered in this work. Because of this small variation, most of the results presented in this work come from a single protein model. Consequently, the accuracy of the resulting mobilities should be about $3 \%$ relative to that of a more exhaustive study in which multiple charge models are considered for every rung of every protein.

In Figures 2-6, mobility is plotted versus $n$ for the five proteins examined in this work. The Yoon and Kim model results (eq 9) are represented by diamonds. The axial ratios of the corresponding prolate ellipsoid are estimated from the crystal structure, as explained in section II.A. The major and minor axes of the ellipsoid are estimated from $D_{\mathrm{t}}$ (eqs 7 and 13). Squares and triangles represent BE model mobilities in the absence and presence of ion relaxation, respectively. Finally, experimental mobilities are represented by $x$ 's. Some overlap

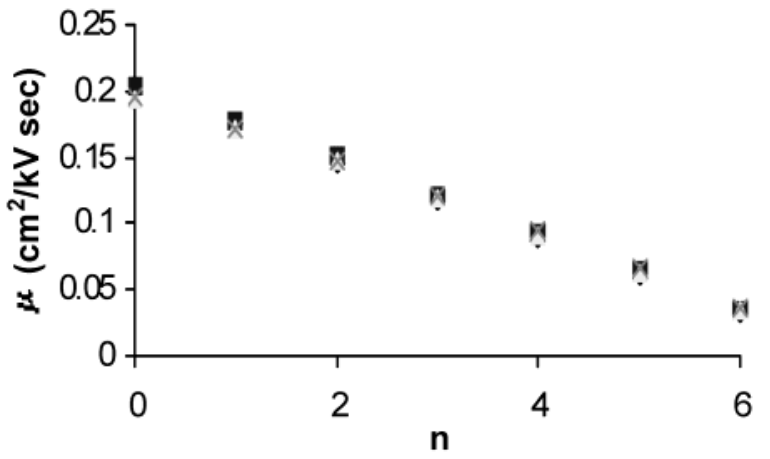

Figure 3. Mobility vs $n$ for hen egg lysozyme. The studies are carried out in $25 \mathrm{mM}$ Tris base plus $192 \mathrm{mM}$ glycine at $25^{\circ} \mathrm{C}$. Yoon and Kim model results are represented with diamonds, $\mathrm{BE}$ in the absence of ion relaxation is represented with squares, $\mathrm{BE}$ including ion relaxation is represented with triangles, and experimental mobilities (7) are represented with $\times$ 's.

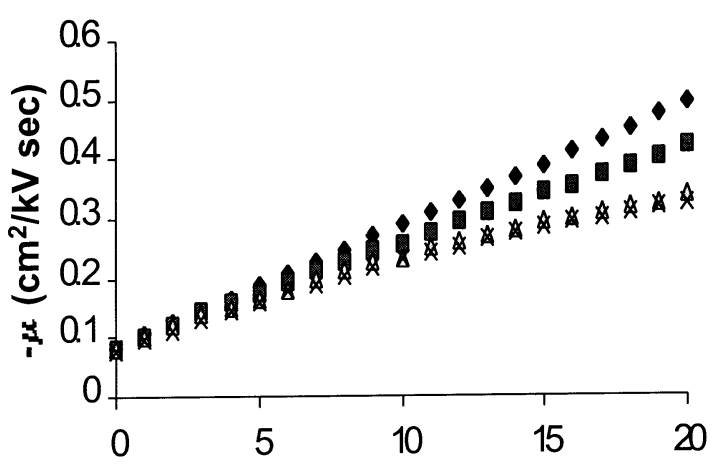

Figure 4. Mobility vs $n$ for bovine superoxide dismutase (SOD) dimer. The studies are carried out in $25 \mathrm{mM}$ Tris base plus $192 \mathrm{mM}$ glycine at $25{ }^{\circ} \mathrm{C}$. Yoon and Kim model results are represented with diamonds, $\mathrm{BE}$ in the absence of ion relaxation is represented with squares, $\mathrm{BE}$ including ion relaxation is represented with triangles, and experimental mobilities (7) are represented with $\times$ 's.

of symbols occurs in Figures 2 and 3. For all five proteins, it is clear that the model results in best agreement with experiment are the BE-with-ion-relaxation values. In the case of lysozyme, $\alpha$-lactalbumin, and SOD, the agreement between experiment and $\mathrm{BE}$ modeling (with ion relaxation) is excellent. It should be emphasized that there are no adjustable parameters in modeling at this point. With the assumptions inherent in the continuum primitive model and the approximate method of predicting charge distribution and net charge, excellent agreement between modeling and experiment may be achieved. The nonlinear behavior in experimental values of $\mu$ that is particularly noticeable in the rungs of the ladder with the largest magnitude of net charge can be attributed to both a breakdown in the validity of the linear PB equation and the contribution of ion relaxation. This is in agreement with previous work using a spherical model of proteins. ${ }^{2}$ For the BE model studies without 


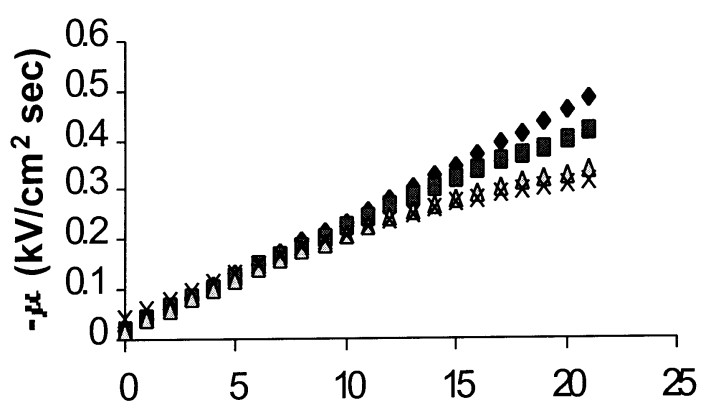

Figure 5. Mobility vs $n$ for human carbonic anhydrase II. The studies are carried out in $25 \mathrm{mM}$ Tris base plus $192 \mathrm{mM}$ glycine at $25^{\circ} \mathrm{C}$. Yoon and Kim model results are represented with diamonds, BE in the absence of ion relaxation is represented with squares, BE including ion relaxation is represented with triangles, and experimental mobilities (7) are represented with $\times$ 's.

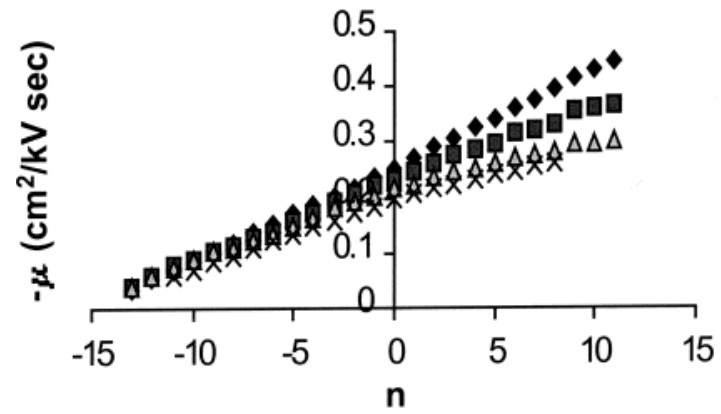

Figure 6. Mobility vs $n$ for hen ovalbumin. The studies are carried out in $25 \mathrm{mM}$ Tris base plus $192 \mathrm{mM}$ glycine at $25^{\circ} \mathrm{C}$. Yoon and Kim model results are represented with diamonds, $\mathrm{BE}$ in the absence of ion relaxation is represented with squares, BE including ion relaxation is represented with triangles, and experimental mobilities (7) are represented with $\times$ 's.

ion relaxation, the equilibrium PB equation is solved at the full (nonlinear) level. This explains why the Yoon and Kim model and $\mathrm{BE}$ (no ion relaxation) mobilities, which agree fairly well with each other for rungs of the charge ladder with low absolute protein charge, $|Z|$, drift apart from each other as $|Z|$ gets large. For rungs with low $|Z|$, all of the model calculations (Yoon and $\mathrm{Kim}, \mathrm{BE}$ without ion relaxation, and BE with ion relaxation) are in fairly good agreement with each other. Thus, detailed characteristics of the protein topography and charge distribution do not influence the values of $\mu$ in a significant way. We conclude that the simpler Yoon and Kim model ${ }^{18}$ should yield fairly accurate values of $\mu$ provided $|Z|$ is small. For colloidal particles, this condition is satisfied if the absolute surface potential does not exceed $25 \mathrm{mV} ; 25$ the results of the present study are consistent with this condition.

The results from models of human carbonic anhydrase II (Figure 5) and hen ovalbumin (Figure 6) are not in as good agreement with experiment as those for the other three proteins. For ovalbumin, this is tentatively attributed to inaccurate estimation of the protein charge. An error in the $\mathrm{p} K_{\mathrm{a} i}$ of one or several charge residues would be expected to propagate through all rungs of the charge ladder and produce, to a first approximation, a constant offset between model and experimental values of $\mu$. However, the slope of this curve, $\mathrm{d} \mu / \mathrm{d} n$, should not be affected by inaccuracies in the estimate of net charge. In Figure $7, \mathrm{~d} \mu / \mathrm{d} n$ is plotted for both experiment and BE modeling (with ion relaxation) for ovalbumin. Neglecting scatter, these data are in good agreement with each other and support the explanation of an error in estimating protein charge in this case.

The discrepancy between experiment and modeling of human carbonic anhydrase II is more complicated. As Figure 8 shows,

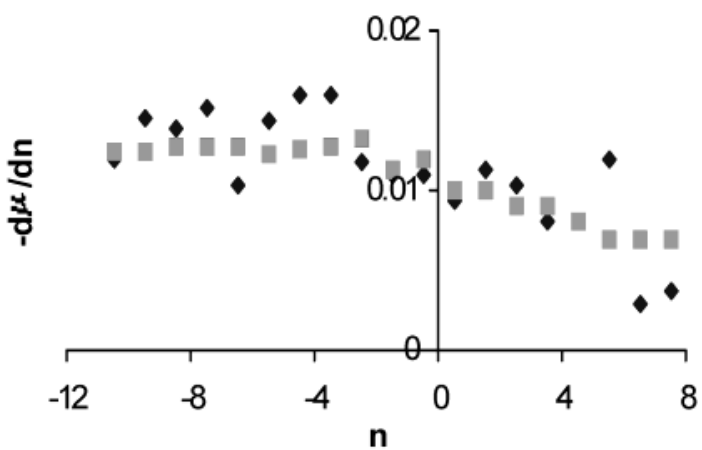

Figure 7. $\mathrm{d} \mu / \mathrm{d} n$ vs $n$ for ovalbumin. Experimental and BE (with ion relaxation) increments are indicated by squares and diamonds, respectively. Conditions are the same as those described in the text and the caption for Figure 6.

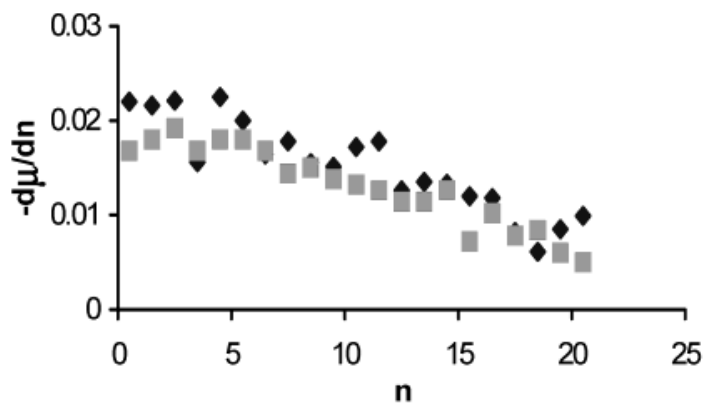

Figure 8. $\mathrm{d} u / \mathrm{d} n$ vs $n$ for human carbonic anhydrase II. Experimental and $\mathrm{BE}$ (with ion relaxation) increments are indicated by squares and diamonds, respectively. Conditions are the same as described in the text and the caption for Figure 5.

TABLE 2: Parameters for Five Proteins

\begin{tabular}{lccc}
\hline \multicolumn{1}{c}{ protein } & $c(\mathrm{~nm})$ & $P$ & $\Delta Z_{\mathrm{BE}}$ \\
\hline$\alpha$-lactalbumin & 1.75 & 0.67 & $-0.99 \pm 0.11$ \\
lysozyme & 1.73 & 0.66 & $-0.97 \pm 0.08$ \\
SOD (dimer) & 2.09 & 0.58 & $-0.92 \pm 0.04$ \\
carbonic anhydrase II & 2.25 & 0.85 & $-0.93 \pm 0.05$ \\
ovalbumin & 2.28 & 0.61 & $-0.86 \pm 0.07(n<0)$ \\
& & & $-0.90 \pm 0.03(n>0)$
\end{tabular}

the slopes, $\mathrm{d} \mu / \mathrm{d} n$, for experiment and BE modeling do not agree with each other as well as in the case of ovalbumin. The slope of the data from experiments is smaller than that from modeling, particularly for small values of $n$. There are two possibilities for this difference in slope. In terms of the Yoon and Kim model, a difference in slope, according to eq 12 , could be due to differences in particle size or $\Delta Z$.

Values of $\Delta Z$ estimated for the five proteins as a function of $n$, following the procedure outlined in section II.D, are summarized in Table 2, together with values of $c$ and $p$ (Yoon and Kim model parameters). We refer to these values as $\Delta Z_{\mathrm{BE}}$ to distinguish them from values determined from electrostatic models, referred to as $\Delta Z_{\mathrm{EM}}$. For human carbonic anhydrase II, $\Delta Z_{\mathrm{BE}}$ does appear to exhibit a small variation with $n$, with $\Delta Z_{\mathrm{BE}}$ $\approx-0.85$ for $n \approx 0$ and approximately -1.0 for large $n$. The scatter in the data makes it impossible to say anything with certainty about the dependence of $\Delta Z_{\mathrm{BE}}$ on $n$. For all of the proteins studied, the values of $\Delta Z_{\mathrm{BE}}$ reported in Table 2 and computed from eq 15 are averages over all rungs of the charge ladder. For hen ovalbumin, values of $\Delta Z_{\mathrm{BE}}$ for the amidation $(n<0)$ and acylation $(n>0)$ portions of the charge ladder are averaged separately.

Because we now have two different ways of estimating $\Delta Z$, values of $\Delta Z_{\mathrm{BE}}$ will be compared with values of $\Delta Z_{\mathrm{EM}}$. The Lee model, described in section II.C, predicts a value of $\Delta Z_{\mathrm{EM}}$ 


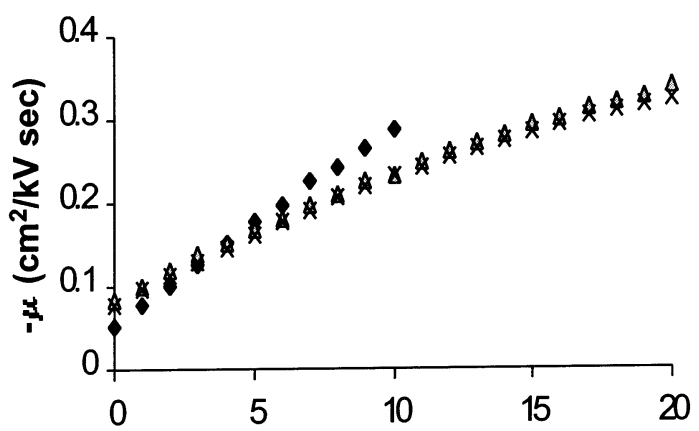

Figure 9. Comparison of BE monomer and dimer mobilities of SOD with experimental mobilities. The diamonds and triangles correspond to the $\mathrm{BE}$ (with ion relaxation) model mobilities of the monomer and the dimer, respectively. Experimental mobilities are denoted by $X$ 's. Conditions are the same as those in Figure 4.

$=-0.997$ for carbonic anhydrase, which indicates that, for this particular protein under these conditions, effects of charge regulation are expected to be small. This result is in contrast with the value of $\Delta Z_{\mathrm{BE}}=-0.93 \pm 0.04$. This value of $\Delta Z_{\mathrm{BE}}$ is, however, in good agreement with the value of $\Delta Z_{\mathrm{EM}}=-0.93$ estimated previously for bovine carbonic anhydrase using the Linderstrom-Lang model. ${ }^{47}$ The accuracy in values of $\Delta Z_{\mathrm{EM}}$ reflects the accuracy with which electrostatic models predict values of $\mathrm{p} K_{\mathrm{a}}$. The accuracy in values of $\Delta Z_{\mathrm{BE}}$ reflects the accuracy of the experiments and of the mobilities predicted by the BE model. Given the known difficulties with predicting accurate values of $\mathrm{p} K_{\mathrm{a}}$ using electrostatic models and the apparent accuracy of the BE model for predicting electrophoretic mobilities, we propose that values of $\Delta Z_{\mathrm{BE}}$ provide a more accurate estimate of the true value of $\Delta Z$ than $\Delta Z_{\mathrm{EM}}$. The weakness of the BE approach is that it assumes the solution structure may be approximated by the model structure.

To address the issue of solution structure in the context of the discrepancy between values of $\mathrm{d} \mu / \mathrm{d} n$ from experiments and models of human carbonic anhydrase II, we consider the effect of particle size on predicted values of mobility. On the basis of the Yoon and Kim model, a smaller value of $|\mathrm{d} \mu / \mathrm{d} n|$ for experiments as compared to model predictions can be explained by a larger actual particle size than that employed in modeling. Changes in conformation, dimerization, or aggregation of the protein could account for such a difference in size.

As an extreme case, consider the effect of complete dimerization on the predicted values of the mobility of charge ladders. In this regard, it is worth considering the charge ladder of SOD, since this protein is known to exist in solution as a dimer. ${ }^{45}$ From Figure 4, the model mobility of the dimeric protein agrees very well with experiment. What if we also consider the model mobility of the monomeric protein? The results are presented in Figure 9, and clearly, the monomeric model does not do as well. For the unmodified protein, $|\mu|_{\text {monomer }}<|\mu|_{\text {dimer }}$ but $\mid \mathrm{d} \mu /$ $\left.\mathrm{d} n\right|_{\text {monomer }}>|\mathrm{d} \mu / \mathrm{d} n|_{\text {dimer. Next, contrast experimental and }}$ modeling (BE with ion relaxation) mobilities for human carbonic anhydrase II in Figure 5. Although the pattern is similar to that seen in Figure 9, the differences between model and experimental mobilities of human carbonic anhydrase II are substantially smaller than the differences seen for monomer and dimer SOD models. Thus, dimerization is not a likely cause for the discrepancy between models and experiments for carbonic anhydrase.

We cannot, however, rule out a small expansion of the actual protein relative to that seen in the crystal structure as the reason for the differences in model and experimental mobilities for human carbonic anhydrase. Using the Yoon and Kim model and assuming $p=0.85$, a prolate ellipsoid with the minor axis equal to $2.78 \mathrm{~nm}$ would match the initial $(n \leq 5)$ experimental slope, $\mathrm{d} \mu / \mathrm{d} n$. This is about $5 \AA$ larger than the value given in Table 2.

\section{Summary}

The free solution electrophoretic mobilities of the charge ladders of five proteins have been modeled within the framework of the continuum primitive model. The analytical Yoon and Kim model ${ }^{18}$ provides a good account of the electrophoretic mobility of a protein provided the absolute net charge is small. In general, it is necessary to go beyond the linear PB equation and include the effects of ion relaxation in order to reproduce experimental mobilities. On the basis of the present work, it is concluded that the continuum primitive model can yield mobilities in excellent agreement with experiment provided the underlying models are realistic representations of the actual solution structure. This can be achieved by numerical boundary element methods.

The models described in this work provide a useful approach for determining the values of $\Delta Z$, the change in net charge of proteins due to chemical modification of charged groups. Knowledge of $\Delta Z$ is essential for the use of protein charge ladders in the quantitative description of the electrostatic properties and interactions of proteins. Previous estimates of $\Delta Z$ were made using simulated titration curves, and their accuracy is limited by the ability to calculate accurate values of $\mathrm{p} K_{\mathrm{a}}$ for titratable groups of protein. In contrast, the values of $\Delta Z$ reported in this work are determined by comparing experimental and calculated values of the change in mobility with the number of modified charge groups. The accuracy of these values of $\Delta Z$ reflects the accuracy of the measured values of mobility and the accuracy of the BE model.

This paper supports the view that the continuum primitive model may be more appropriate for the modeling of electrokinetics than for that of electrostatics. Other investigators ${ }^{1-15}$ have examined the ability of the equilibrium CPM to predict the $\mathrm{p} K_{\mathrm{a}}$ charge residues of proteins, and some discrepancies between modeling and experiment have been observed. As discussed in the Results section, discrepancies in regard to electrokinetic modeling may very well be due entirely to inaccuracies in estimating the net charge of the model particle. In this view, the main challenges to the accurate predictions of electrophoretic mobilities lie primarily in the modeling of electrostatics, not electrokinetics.

It is hoped that this work will stimulate the use of charge ladder experiments on proteins, biomolecules, and biomolecular complexes, coupled with detailed modeling, to address quantitative questions regarding solution structure and charge. Currently, such studies are underway on calmodulin and its complexes as well as the membrane protein lipase and the complex it forms with colipase.

Acknowledgment. J.D.C. would like to thank the Camille and Henry Dreyfus Foundation and the National Science Foundation (Contract CTS-0134429) for financial support.

\section{References and Notes}

(1) Gao, J.; Gomez, F. A.; Harter, R.; Whitesides, G. M. Proc. Natl. Acad. Sci. U.S.A. 1994, 91, 12027.

(2) Carbeck, J. D.; Negin, R. S. J. Am. Chem. Soc. 2001, 123, 1252.

(3) Gao, J.; Whitesides, G. M. Anal. Chem. 1997, 69, 575.

(4) Gao, J. M.; Mrksich, M.; Gomez, F. A.; Whitesides, G. M. Anal. Chem. 1995, 67, 3093. 

1370.

(5) Cordova, E.; Gao, J. M.; Whitesides, G. M. Anal. Chem. 1997, 69

(6) Negin, R. S.; Carbeck, J. D. J. Am. Chem. Soc. 2002, 124, 2911.

(7) Carbeck, J. D.; Colton, I. J.; Anderson, J. R.; Deutch, J. M.; Whitesides, G. M. J. Am. Chem. Soc. 1999, 121, 10671.

(8) Henry, D. C. Proc. R. Soc. London, Ser. A 1931, A33, 106.

(9) Menon, M. K.; Zydney, A. L. Anal. Chem. 2000, 72, 5714.

(10) Linderstrom-Lang, K. C. Trav. Lab. Carlsberg 1924, 15, 1.

(11) Bashford, D.; Karplus, M. Biochemistry 1990, 29, 10219.

(12) Yang, A.-S.; Gunner, M. R.; Sampogna, R.; Sharp, K.; Honig, B. Proteins 1993, 15, 252.

(13) Antosiewicz, J.; Briggs, J. M.; Elcock, A. H.; Gilson, M. K.; McCammon, J. A. J. Comput. Chem. 1996, 17, 1633.

(14) Zhou, H.-X.; Vijayakumar, M. J. Mol. Biol. 1997, 267, 1002.

(15) Grycuk, T. J. Phys. Chem. B 2002, 106, 1434.

(16) Caravella, J. A.; Carbeck, J. D.; Duffy, D. C.; Whitesides, G. M. Tidor, B. J. Am. Chem. Soc. 1999, 121, 4340.

(17) Sharma, U.; Negin, R. S.; Carbeck, J. D. J. Phys. Chem. B 2003 107, 4653.

(18) Yoon, B. J.; Kim, S. J. Colloid Interface Sci. 1989, 128, 275.

(19) Allison, S. A. Macromolecules 1996, 29, 7391

(20) Allison, S. A. Biophys. Chem. 2001, 93, 197.

(21) Allison, S. A.; Wall, S.; Rasmusson, M. J. Colloid Interface Sci. $\mathbf{2 0 0 3}, 263,84$.

(22) Overbeek, J. T. G. Kolloid-Beih. 1943, 54, 287.

(23) Booth, F. Proc. R. Soc. London, Ser. A 1950, 203, 514

(24) McQuarrie, D. A. Statistical Mechanics; Harper \& Row: New York, 1976; Chapter 15

(25) Hunter, R. J. Introduction to Modern Colloid Science; Oxford Science Publications: New York, 1993; p 239.

(26) Handbook of Biochemistry: Selected Data for Molecular Biology, 2nd ed.; Sober, H. A., Ed.; The Chemical Rubber Company: Cleveland, $\mathrm{OH}, 1970$; Section C.

(27) Cantor, C. R.; Schimmel, P. R. Biophysical Chemistry, Part II; W.
H. Freeman \& Co.: San Francisco, CA, 1980; Chapter 10.

(28) Marion, J. B. Classical Dynamics of Particles and Systems; Academic Press: New York, 1970; Chapter 12.

(29) Goldstein, H. Classical Mechanics; Addison-Wesley: Reading, MA, 1950; Chapter 5.

(30) Fair, M. C.; Anderson, J. L. J. Colloid Interface Sci. 1989, 158, 388 .

(31) Wiersema, P. H.; Loeb, A. L.; Overbeek, J. T. G. J. Colloid Interface Sci. 1965, 22, 78 .

(32) O'Brien, R. W.; White, L. R. J. Chem. Soc., Faraday Trans. 2 1978, 74, 1607

(33) Stigter, D. J. Phys. Chem. 1978, 82, 1417.

(34) Stigter, D. J. Phys. Chem. 1978, 82, 1424. 100 .

(36) Wilson, W. W.; Salin, M. W. Mol. Cell. Biochem. 1981, 36, 157.

(37) Allison, S. A.; Mazur, S. Biopolymers 1998, 46, 359.

(38) Allison, S. A. Macromolocules 1998, 31, 4464.

(39) Kuramitsu, S.; Hamaguchi, K. J. Biochem. (Tokyo) 1980, 87, 1215

(40) Bartik, K.; Redfield, C.; Dobson, C. M. Biophys. J. 1994, 66, 1180.

(41) Anderson, D. E. Ph.D. Dissertation, University of Oregon, Eugene, OR, 1992

(42) Allison, S. A.; Potter, M.; McCammon, J. A. Biophys. J. 1997, 73, 33.

(43) Durant, J. A.; Chen, C.; Laue, T. M.; Moody, T. P.; Allison, S. A. Biophys. J. 2002, 101-102, 593

(44) Lee, L. K.; Fitch, C. A.; Garcia-Moreno, B. E. Protein Sci. 2002 11,1004 .

(45) Cudd, A.; Fridovich, I. J. Biol. Chem. 1982, 257, 11443.

(46) Stein, P. E.; Leslie, A. G. W.; Finch, J. T.; Carrell, R. W. J. Mol. Biol. 1991, 221, 941 .

(47) Gitlin, I.; Mayer, M.; Whitesides, G. M. J. Phys. Chem. B 2003, 107, 1466. 